\title{
Neon Gas Field Ion Source - Stability and Lifetime
}

\author{
John Notte, ${ }^{*}$ FHM Faridur Rahman, ${ }^{*}$, Shawn McVey, ${ }^{*}$ Shida Tan, ${ }^{* *}$ and Richard H. Livengood** \\ * Carl Zeiss SMT Inc, One Corporation Way, Peabody, MA 01960 \\ ** Intel Corporation, MS: SC9-68, Santa Clara, CA 95054
}

Soon after its development in 1955, the gas field ion source (GFIS) was pursued as the source of positive ions for focused ion beam (FIB) instruments [1]. Within the semiconductor industry, such FIB instruments are of critical importance for their failure analysis (FA), circuit edit (CE), and TEM sample preparation. However the GFIS development efforts were hampered by issues related to the source lifetime, and the short and long term temporal stability. The commercial gallium liquid metal ion source (Ga-LMIS) has served as the ion source of choice for the past 30 years with some recognized shortcomings arising from the probe size, electrical contamination, optical opacity, etc [2]. These shortcomings have produced a growing interest in FIBs with other ion species. In the past decade, the helium GFIS performance was vastly advanced - permitting the development of the helium ion microscope (HIM). In the past year, these same advances were applied to a neon GFIS.

The challenges arising from a neon beam can be attributed to the fundamentally different characteristics of the neon atom compared to a helium atom. Neon atoms will field ionize at an electric field strength of $3.5 \mathrm{~V} / \AA$, or about $20 \%$ less than the field required for the field ionization of helium[3]. The reduced field strength gives rise to a limited lifetime of the emitter atoms due to field assisted chemical processes. Previous neon results show drastic changes in the apex of the emitter after typically a few minutes of operation [3]. The neon gas also is much more polarizable in the presence of a strong non-uniform electric field. This has the effect of making the gas atoms attracted to the emitter, and augmenting their normally small van der Waals forces to the point where they tend to adhere to the emitter [4]. As an indirect consequence, the emission current tends to fluctuateas muh as 30\% over very short time periods (Figure 1a). Additionally, the location of the emission sites tends to jump between a small set of metastable states (Figure 1b).

Most recently, the stability and lifetime of the neon GFIS has been improved to the point where it has become a suitable source for a neon ion microscope (NIM). Both the fluctuations in the brightness, and the motion of the emission sites, have been reduced by a factor of 5 . Further improvements allow for the operation over long periods of time ( $\sim 10$ hours). Under these conditions, the NIM can be used for imaging (Fig. 2) and nano-machining applications.

[1] A.J. Melmed, Applied Surface Science, 94/95 (1996) 17.

[2] J. Orloff, et al., J. Vac Sci. Technol. B., 14(6) (1996) 3759.

[3] E. Müller, et al., Field Ion Microscopy, Elsevier, New York, 1969.

[4] T. Sakurai, et al., Phys. Rev. B., 10(10) (1974) 4205. 
(a) Beam Brightness Profile

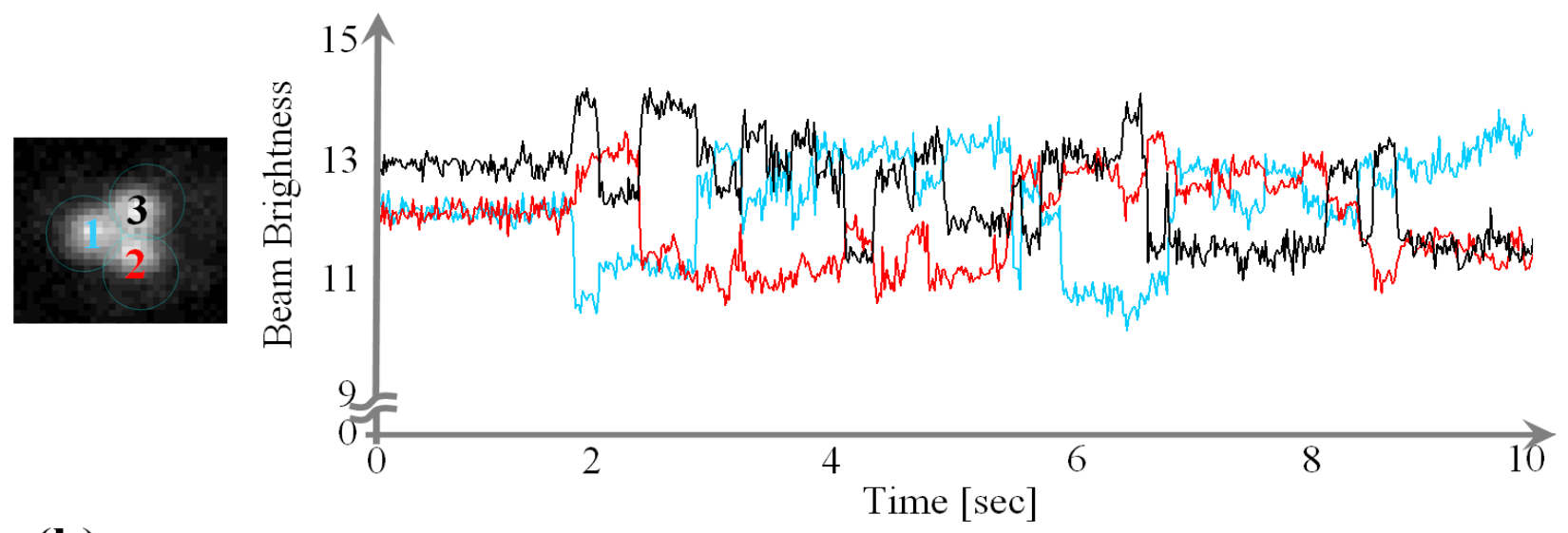

(b) Site Swapping: Centroid of Brightness

b1)

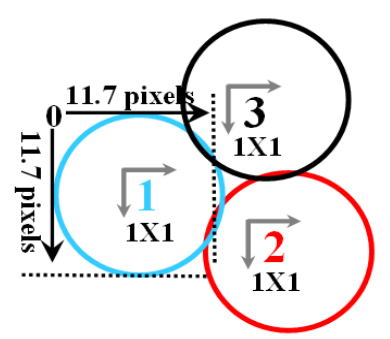

b2)

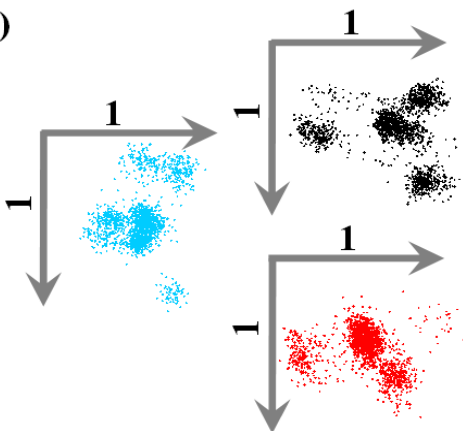

Figure 1: Upper panel shows a FIM image of a built-up tri-atomic (Trimer) ion source achieved using Ne gas with an applied field of about $3.5 \mathrm{~V} / A$. The upper graph shows the beam brightness as a function of time for $1^{\text {st }}, 2^{\text {nd }}$ and $3^{\text {rd }}$ atoms of the trimer (aqua, red, and black). The lower panel shows the locations of the computed brightness centroid for the three atoms.

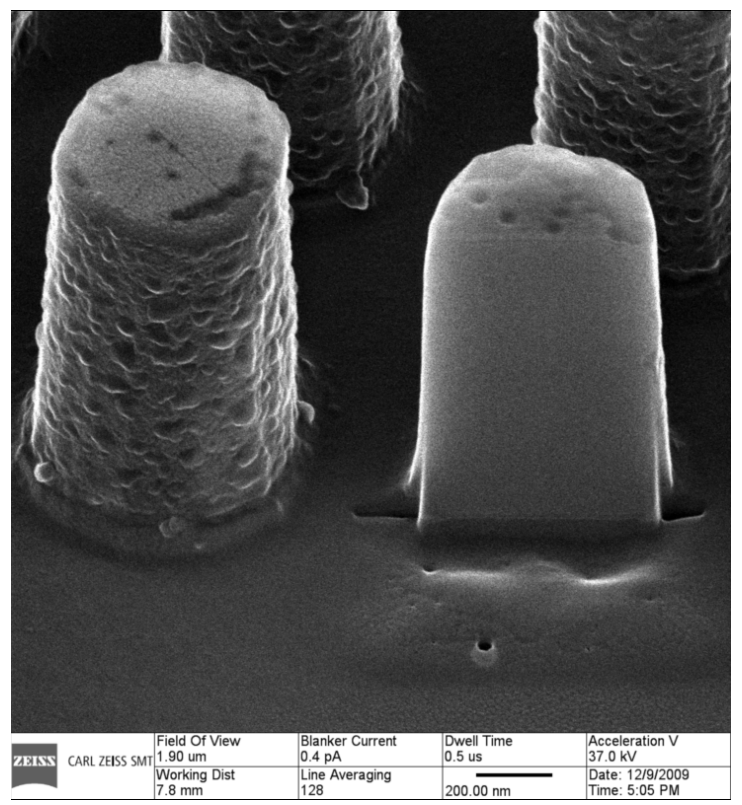

Figure 2: An aluminum post was cross sectioned with a focused neon beam, and then subsequently imaged with the same neon beam. The field of view is $1.9 \mathrm{um}$. 